Motor command inhibition and the representation of response mode during motor imagery

In press, Acta Psychologica

Juliane Scheil ${ }^{1}$ and Baptist Liefooghe ${ }^{2}$

${ }^{1}$ Leibniz Research Centre for Working Environment and Human Factors

${ }^{2}$ Department of Experimental-Clinical \& Health Psychology, Ghent University

Correspondence concerning this paper should be addressed to Juliane Scheil, Leibniz Research Centre for Working Environment and Human Factors, Ardeystraße 67, 44139 Dortmund, Germany, Tel. ++49 (0)231 1084-313, Fax ++49 (0)231 1084-340, e-mail: scheil@ifado.de.

Acknowledgements:

Juliane Scheil was supported by a scholarship of the German Academic Exchange Service (DAAD). Baptist Liefooghe is supported by grant BOF16/MET_V/002 of Ghent University and grant G009517N by the Flemish Government. The authors are indebted to Jan De Houwer for his support. 


\begin{abstract}
Research on motor imagery proposes that overt actions during motor imagery can be avoided by proactively signaling subthreshold motor commands to the effectors and by invoking motorcommand inhibition. A recent study by Rieger, Dahm, and Koch (2017) found evidence in support of motor command inhibition, which indicates that $\mathrm{MI}$ cannot be completed on the sole basis of subthreshold motor commands. However, during motor imagery, participants know in advance when a covert response is to be made and it is thus surprising such additional motor-command inhibition is needed. Accordingly, the present study tested whether the demand to perform an action covertly can be proactively integrated by investigating the formation of task-specific action rules during motor imagery. These task-specific action rules relate the decision rules of a task to the mode in which these rules need to be applied (e.g., if smaller than 5, press the left key covertly). To this end, an experiment was designed in which participants had to switch between two numerical judgement tasks and two response modes: covert responding and overt responding. First, we observed markers of motor command inhibition and replicated the findings of Rieger and colleagues. Second, we observed evidence suggesting that task-specific action rules are created for the overt response mode (e.g., if smaller than 5, press the left key). In contrast, for the covert response mode, no task-specific action rules are formed and decision rules do not include mode-specific information (e.g., if smaller than 5, left).
\end{abstract}

Keywords: Task switching; motor imagery; inhibition 


\section{Motor command inhibition and the representation of response mode during motor imagery}

\section{Introduction}

Motor imagery (MI) refers to a dynamic state during which the representation of a given motor act is internally rehearsed within working memory without any overt movement (Decety \& Grezes, 1999, p. 177; see also Collet \& Guillot, 2010; Moran et al., 2012). MI-based techniques are widely used for optimizing a variety of complex skills such as in athletics (see Weinberg, 2008, for a review), typing (Nyberg, Eriksson, Larsson, \& Marklund, 2006), playing music (e.g., Highben \& Palmer 2004; Lim \& Lippman, 1991), or even surgical interventions (e.g., Rogers, 2006). A key proposal of MI is the Motor Simulation Theory (MST) developed by Jeannerod $(1994,2001,2006)$. MST is based on the assumption that $\mathrm{MI}$ includes motor representations, which are also involved in the preparation and initiation of actual movements (Jeannerod, 1994). This stance is evidenced by a substantial body of neuro-physiological research, which indicate that the neural circuitry underlying $\mathrm{MI}$ partially overlaps with the neural circuitry underlying the overt execution of actions (cf. Burianová et al., 2013; Kraeutner, Gionfriddo, Bardouille, \& Boe, 2014). In addition, mental-chronometry procedures have repeatedly demonstrated a functional similarity between covert and overt action, namely the time needed to perform a particular action covertly co-varies with the time needed to execute an action overtly (i.e., isochrony, see Guillot et al., 2012b, McAvinue \& Robertson, 2008, for reviews). For instance, Decety, Jeannerod, and Prablanc (1989) observed that increasing the length of a particular walking distance not only increases the actual walking time but also the imagined walking time.

Following MST, motor representations common to overt and covert action are activated through a simulation mechanism during MI (Jeannerod, 2001, 2006). Yet, whereas MI relies on the same representations as the overt action it simulates, the overt action (i.e., actual execution) itself is absent (Jeannerod, 2004, 2006). MI thus constitutes a paradox in which motor representations are activated, on the one hand, and overt movements are avoided, on the other hand (see Jeannerod, 2001, p. S106; Guillot et al., 2012a, p. 2 for a similar argument). Jeannerod (2001) proposed two 
mechanisms through which overt actions are avoided. First, motor activation during $\mathrm{Ml}$ is subliminal, such that it is insufficient to instantiate overt action. Second, motor output during MI is blocked by an inhibitory process, which is generated in parallel with motor activation. Similarly, Guillot et al. (2012b) provided a review on research focusing on motor inhibition during $\mathrm{MI}$ and proposed that overt actions during $\mathrm{MI}$ are avoided in three complementary ways : (a) the demand to perform covert responses is part of the imagery experience and integrated within the construction of the mental representation of the imagined action, such that only subthreshold motor commands are signaled to the effectors; (b) motor commands specific for the effector used in the imagined condition are weakened by inhibitory cerebral regions, possibly complemented with cerebellar and spinal inhibitory influences (i.e. effector-specific motor-command inhibition); and (c) all motor activity is inhibited (global motor-command inhibition).

As pointed by Guillot et al. (2012b, p. 8), motor-command inhibition during MI shows parallels with a well-documented phenomenon in cognitive psychology, namely response inhibition in which participants are required to withhold an overt response when a specific signal is presented (see Verbruggen \& Logan, 2008, 2009 for reviews). A common finding in research on response inhibition is that trials following a trial on which a response was inhibited are responded to much more slowly, which has been attributed to residual inhibitory processing (e.g., Rieger \& Gauggel, 1999; Verbruggen, Logan, Liefooghe, \& Vandierendonck, 2008). Based on this type of findings, Rieger and colleagues (2017) investigated the processes underlying MI by comparing aftereffects of overt and covert responding (i.e., MI). To this end, they developed a novel response-mode switching paradigm. A computer display presented four horizontally aligned empty circles, which corresponded with four horizontally aligned response keys. When a circle was filled, participants had to press the corresponding response key. For the two leftwards circles, the left hand had to be used. For the two rightwards circles, the right hand had to be used. Depending on the color of the filled circle, participants responded to a trial either overtly or covertly. More precisely, participants had to release a rest-key and press the corresponding response key overtly or covertly before returning to the rest 
key. Such procedure provides two dependent measures, which are common to overt and covert responding: Release Times (the time between stimulus onset and releasing the rest-key) and Inter Rest-Key Intervals (the time interval between releasing the rest-key and re-entering the rest-key). In the condition of interest, the mode repeated or switched on a trial-by-trial basis and four trial sequences were created: $C$ (covert trial $n-1)-O$ (overt trial $n$ ) sequences; O-O sequences; O-C sequences and $\mathrm{C}-\mathrm{C}$ sequences. Release Times were longer for $\mathrm{C}-\mathrm{O}$ sequences than for $\mathrm{O}-\mathrm{O}$ sequences. Switching from the covert to the overt mode thus elicited a mode switch cost. The difference between $\mathrm{C}-\mathrm{C}$ and $\mathrm{O}-\mathrm{C}$ sequences was not significant. Inter Rest-Key Intervals were longer for $\mathrm{C}-\mathrm{C}$ sequences compared to $\mathrm{O}-\mathrm{C}$ sequences. A mode switch benefit was thus observed. Inter Rest-Key Intervals did not differ significantly between $\mathrm{O}-\mathrm{O}$ and C-O sequences. Rieger et al. (2017) concluded that the aftereffects of $\mathrm{Ml}$ they observed provided evidence for global motor-command inhibition. This inhibition persists over time and affects performance on trial $n$. Avoiding an overt response on trial n-1 impairs the motor commands involved in executing an overt response on trial $n$, but also the motor commands involved in releasing and re-entering the rest-key. Additional analyses were also conducted in which the repetition of specific hand movements across two trials was taken into account (i.e., hand repetition vs. hand alternation). Hand repetitions were slower than hand alternations in the $\mathrm{C}-\mathrm{C}$ and $\mathrm{C}-\mathrm{O}$ sequences, whereas the reverse pattern was observed for the $\mathrm{O}-\mathrm{O}$ sequences. Rieger et al. (2017) interpreted this pattern as evidence for the hypothesis that MI is also associated with effector-specific motor-command inhibition, which hampers the repetition of the same effector across two trials. Interestingly, Rieger et al. (2017) also compared complete repetitions (i.e., same hand, same stimulus) with partial repetitions (i.e., same hand, different stimulus), but did not observe differences between both, which led to the conclusion that MI does not involve actionspecific motor-command inhibition.

The findings of Rieger et al. (2017) stress the importance of global and effector-specific motor command inhibition during $\mathrm{MI}$ and the specific pattern of after-effects they observed is difficult to reconcile with the idea that $\mathrm{MI}$ is solely based on subthreshold commands, without 
invoking additional motor-command inhibition. However, this does not exclude that motor commands during $\mathrm{MI}$ may be weaker compared to actual response execution, nor that the demand to perform a task covertly is somehow proactively integrated with the other features of task. As pointed out by Guillot et al. (2012a, p. 8), when participants engage in MI they know in advance that only a covert movement is required and it would be puzzling that overt movements during $\mathrm{MI}$ can only be avoided through motor command inhibition. In analogy, research on response inhibition recently demonstrated that response inhibition also depends on preparatory processes, which proactively adjust the necessary attentional and response parameters in view of the future requirement to inhibit a response when needed (Elchlepp, Lavric, Chambers, \& Verbruggen, 2016; Verbruggen, Stevens, \& Chambers, 2014). This state of affairs raises the question to which extent the demand to respond covertly can be integrated proactively with the other features of an imagined movement, when signaled that MI ensues. In the present study, we tested this issue by investigating whether task-specific action rules are created during MI. These rules integrate the decision rules of a particular task (e.g., odd-left, even-right) with the parameters specifying the response modality in which these decisions rules need to be applied (e.g., saying the word left, pressing a left key; Philipp $\&$ Koch, 2010). The central question was if such task-specific action rules are created when signaled that a task needs to be performed covertly (i.e., MI), such as 'if smaller than 5, press the left key covertly". To this end, the procedure of Rieger et al. (2017) was extended to also incorporate task switches besides response-mode switches.

Task switching has been widely used in order to investigate how different task parameters are represented and configured (see Kiesel et al., 2010; Monsell, 2003; Vandierendonck, Liefooghe, \& Verbruggen, 2010, for reviews). A core observation in task switching is the task-switch cost, which manifests itself as higher reaction times and error rates when the task switches compared with repeating the same task as in trial $n-1$. The switch cost offers a marker of the processes involved in configuring the cognitive system towards a new task, which prepare for that task (e.g., Rogers \& Monsell, 1995) and shield the cognitive system from proactive interference emanating from previously executed tasks (e.g., Dreisbach \& Haider, 2009; see also Vandierendonck et al., 2010, for 
an extensive review of the interplay between preparation and interference in task switching). The task-switch cost has been frequently used as a marker to measure how different task parameters are organized when performing a task (e.g., Allport, Styles, \& Hsieh, 1994; Hübner, Futterer, \& Steinhauser, 2001; Kleinsorge \& Heuer, 1999; Phillip \& Koch, 2010; Vandierendonck, Christiaens, \& Liefooghe, 2008). These parameters can be thought of as cognitive representations of the different components of a task, such as the goal of that task, the stimulus-response categorization rules needed to achieve the task goal, the response modality, the relevant stimulus dimension, and so on. Previous research demonstrated that in some cases task parameters are integrated into a single representation underlying task performance, which is commonly labelled the task-set (Rogers \& Monsell, 1995). Evidence for this hypothesis comes from task-switching studies in which not only tasks can repeat or switch, but also the relevant stimulus dimension (Vandierendonck et al., 2008) or the response modality (Hübner et al., 2001; Kleinsorge \& Heuer, 1999; Philipp \& Koch, 2010). Philipp and Koch (2010) used a task-switching design in which participants had to switch between two tasks (e.g., parity and magnitude judgments) and two overt response modalities (verbal, manual). They observed that the cost of switching both the response modality and the task at the same time was almost similar to the cost of changing the response modality (modality switching) or the task (task switching) separately. Such under-additive switch-pattern (i.e., cost of changing two components < cost of changing component $1+$ cost changing component 2 ) indicates that response modality is integrated with the other task parameters into a single task-set. Whenever the response modality, the task, or both are changed, the entire task-set is rebuilt. Philipp and Koch (2010) proposed that in this situation task-specific action rules are created, which incorporate both the response modality and the decision rules of that task (i.e., the category-response mappings). For instance, for the magnitude task in the verbal modality these action rules are "if smaller than 5, say left" and "if larger than 5 , say right", whereas for the manual modality these action rules are "if smaller than 5 , press the left-key" and "if larger than 5, press the right-key." Nevertheless, task parameters are not always integrated into a single task-set. For instance, Hübner et al. (2001) observed that switching two task components led to a cost, which was almost equal to the sum of the costs associated with changing 
each component separately. Such additive switch pattern (i.e., cost of changing two components = cost of changing component $1+$ cost of changing component 2) indicates that each component can be configured separately without affecting the configuration of other components. When two components are changed, reconfiguration of each component occurs in a linear fashion, with each reconfiguration cost adding up to an overall performance cost (Vandierendonck et al., 2008).

Based on the task-switching approach outlined in the previous paragraph, we investigated whether task-specific action rules are created during motor imagery, such as "if smaller than 5 , press the right key covertly" and "if larger than 5, press the left key covertly"? To this end, we conducted an experiment in which participants switched between two tasks, on which they could either respond overtly or covertly. Each task required either a left or a right key-press. For both tasks, responses were only performed with the index finger of the right hand and performance was measured by using Release Times and Inter Rest-Key Intervals. First, we measured indices of motor-command inhibition. To this end, we focused on the interaction between response mode (overt, covert) and responsemode transition (mode repetition, mode switch), as was previously done by Rieger et al. (2017). The presence of motor-command inhibition could be inferred by the presence of response-mode switch costs for the overt response mode and response-mode switch benefits for the covert response mode. Note that, because we only used one effector, we could not dissociate whether such pattern reflects global or effector-specific motor command inhibition, which was not the aim of the current study. In view of the results of Rieger et al. (2017), we will refer to the term global/effector-specific motor command inhibition in the remainder of the text. However, our procedure allowed us to further investigate the role of action-specific inhibition in MI. To this end, we focused on response-repetition effects, which refers to the common finding that response repetitions are faster than response switches (e.g., Kleinsorge, 1999). Because only one effector was used in the present study (i.e., the right hand), response repetitions are action repetitions and response alternations are action switches. Following the conclusion of Rieger et al. (2017) that action-specific inhibition does not play a role in $\mathrm{Ml}$, response repetition and response alternations should be disrupted to the same extent following MI. Alternatively, if MI does involve action-specific inhibition, then response repetitions 
should be disrupted to a larger extent after MI compared to response alternations, thus leading to a reduction or reversal of the response-repetition effect.

Second, we investigated if task-specific action rules were created by investigating the interaction between response-mode transition and task transition (task repetition vs. task switch). For the overt response mode, the results of Philipp and Koch (2010) suggest that task-specific action rules are created and we expected an under-additive switch pattern, indicating that the cost of changing both the task of and the response mode is in the same range as changing each component separately. For the covert response mode, two scenarios are possible. On the one hand, the prospective nature of $\mathrm{MI}$ (Guillot et al., 2012b) and the importance of preparatory processing in response inhibition (Elchlepp et al., 2016; Verbruggen et al., 2014), suggest that the covert response mode can be integrated with other task parameters. Accordingly, a sub-additive switch pattern would be expected, indicating the creation of task-specific action rules in which the demand to respond covertly is included. On the other hand, the results of Rieger et al. (2017) indicated that $\mathrm{MI}$ is not associated with subthreshold motor commands and the possibility remains the even if participants are requested to perform $\mathrm{Ml}$, task-specific action rules are not created, thus predicting an additive switch pattern.

\section{Material and Methods}

\subsection{Participants}

A sample of 60 right-handed volunteers were recruited at Ghent University (47 females, mean age $=23.15$ years). All participants signed an informed consent, which was approved by the Ethical Committee of Ghent University. They were payed 10 euros for their participation.

\subsection{Stimuli, tasks, and apparatus}

Stimuli consisted of the digits 1-9 (excluding 5). Each numerical judgement task was cued by one of two different cues and cue repetitions were avoided (see Monsell \& Mizon, 2006). Digits had 
to be classified either as smaller or larger than 5, when the cue "KLEIN-GROOT" (Dutch for smalllarge) or the cue "K-G" appeared above the target stimulus. When the cue "ONEVEN-EVEN" (oddeven) or "O-E" appeared, participants had to decide whether the number was odd or even. All tasks and task cues occurred with equal frequency. The covert and overt mode was also cued by using two cues per mode, without cue repetitions. The demand to respond overtly was indicated by either the cue "VOER UIT" (execute in Dutch) or "FYSIEK" (physical in Dutch). The demand to respond covertly was indicated by the cue "BEELD IN" (imagine in Dutch) or "MENTAAL" (mentally in Dutch). All variables (tasks, task transition, mode, mode transition, responses, and cues) were pseudorandomized across experimental subsets of four blocks. Cues and stimuli were presented in white on a black background one below the other, whereby the imperative stimulus was always below the cues. For half the subjects, the task cue appeared above the mode cue, whereas the reverse was true for the other half. Viewing distance was approximated $60 \mathrm{~cm}$. Participants pressed a left-key (the "A"-key of an AZERTY keyboard) as a response to small and uneven digits and a right-key (the " $\mathrm{P}$ "key) for large and even digits. The spacebar served as start key.

\subsection{Design and Procedure}

After giving informed consent, participants were outlined the general purpose of the experiment, which was described as measuring the power of imagination. Next, on-screen instructions specified how participants had to respond overtly to both tasks (i.e., the use of the release key) and the task rules of both tasks. Afterwards, the instructions for the covert trials were provided. These instructions were virtually the same, for the exception that participants were now told that they should imagine responding to each task. More specifically, they were told to release the rest key and imagine pressing one of the two response keys without actually doing it. To encourage participants to engage in $\mathrm{Ml}$, an electrode was connected to their right index finger, which was attached to a bogus device. Participants were told that we were able to measure the degree to which they performed MI. In addition, a fake calibration phase was conducted in which the bogus 
device was tested. All responses were made with the right index finger. The experiment was run in a single session that took about 60 minutes.

For creating balanced task sequences, four factors were considered: Task Transition, Mode Transition, Response Transitions, and Task-Rule Congruency. Each factor contains two levels, resulting in 16 combinations. In order to create balanced sequences in which each combination follows upon another combination an equal number of times, a balanced randomization function was used which requires $16 \times 16+1=257$ trials. Three of these massive blocks were administered during the experiment. However, because these blocks were far too long for the participants they were divided in four smaller blocks. The first block contained 65 trials. The second block started with the presentation of the last trial of the previous block and again consisted of 65 trials. For the third and the fourth block the same procedure was used. Accordingly, the whole experiment consisted of 12 blocks of 65 trials each. The first trial of each block was removed from analyses, resulting in 64 trials that were available for analyses (i.e., 768 in total). For the practice block, a virtual block of 257 trials was also created, but participants only performed the first 50 trials of that sequence.

Each trial was initiated by participants pressing the spacebar. If the spacebar was not already pressed at the beginning of the trial, the request "SPATIE" (spacebar in Dutch) was presented in the center of the screen until pressing of the spacebar or until a maximum time of $3,000 \mathrm{~ms}$ had elapsed. If the spacebar was already pressed at the beginning of the trial, this display was not presented. When the spacebar was pressed, a fixation cross was displayed for $1,000 \mathrm{~ms}$, followed by the task cue, mode cue, and imperative stimulus that remained visible until participants pressed the spacebar again to end the trial or until 3,000ms had elapsed. On the overt trials, participants were required to release the spacebar, press the response key and return to the spacebar. On the covert trials, the spacebar had to be released. After that, participants had to imagine the movement without actually moving the finger towards the response keys. The spacebar had to be pressed when the imagined movement was finished. Pressing the spacebar again terminated the trial and initiated the next one. At the end of each block, participants received error feedback. To simulate registration of imagined 
movements with the bogus device, the amount of errors made on the overt trials was doubled for the feedback (e.g., if a participant made two errors in the overt condition, the error amount in the feedback was four).

\subsection{Data analysis}

Two participants had to be excluded because of not following the instructions. Based on the absolute deviation around the median (Leys, Ley, Klein, Bernard, \& Licata, 2013), participants with a median error rate above $12.74 \%$ were excluded, which led to an additional loss of six participants ${ }^{1}$. The final sample consisted of 12 men and 40 women with a mean age of 23.04 years (range: 18-28). The practice phase was excluded from the analyses, as was the first trial of each block. In addition, invalid trials (in which the spacebar was not released or not pressed again, or in which a key was pressed that did not belong to the set of response keys, $3.4 \%$ in total) were discarded. Of main interest were the Release Times and the IRKIs, which are common to overt and covert trials. For your guidance, a schematic illustration of both measures is also presented in Figure 1. Because error data was not available in the covert trials, errors were not discarded for the overt trials.

Figure 1 about here

Several analyses were conducted on each dependent measure. First, in order to check whether participants did perform MI on the covert trials, the correlation between the RT on the covert trials and the RT on the overt trials was calculated (see Guillot et al., 2012b for a discussion of this approach). Of course, one could argue that this is no evidence for participants really engaging in MI but instead, it could merely suggest that participants just waited the estimated amount of time they would need to move to the response key before going back to the spacebar and this without actually imagining doing this movement. To account for this possibility, we also checked whether response-congruency effects (Sudevan \& Taylor, 1987) were present on the covert trials. Responsecongruency effects are caused by stimuli affording the same (congruent stimulus) versus different

\footnotetext{
${ }^{1}$ Please note that the data patterns of main interest were not changed in analyses including all participants.
} 
responses (incongruent stimulus) across both tasks. Typically, incongruent stimuli are responded to more slowly than congruent stimuli (see Meiran \& Kessler, 2008 for a review).

In a second step, we investigated the presence of global/effector-specific motor command inhibition and whether the covert response mode is integrated with other task parameters. To this end, a repeated measures ANOVA was conducted with the factors Task Transition (task repetition vs. task switch), Mode (overt vs. covert), and Mode Transition (mode repetition vs. mode switch). All post-hoc comparisons were Bonferroni corrected. Third, we investigated the presence of actionspecific inhibition by focusing on response-repetition effects. For this purpose, a repeated measures ANOVA with the within-subjects factors Mode (overt vs. covert), Mode Transition (mode repetition vs. mode switch), and Response Transition (repetition vs. switch) was conducted. For these analyses, invalid trials not only in the current but also in the previous trial were excluded to prevent the factor Response Transition from being contaminated.

In a final step, we also focused on the actual Reaction Times (i.e., the interval from the presentation of the imperative stimulus until pressing of a response key) and Error Rates on the overt trials. In line with more common research on task switching, error trials as well as post-error trials were discarded for the Reaction-Time analysis. Post-error trials were also discarded for the analysis of the Error Rates. Means and standard deviations of both measures are presented in Table 1. Both measures were subjected to a repeated measures ANOVA with the factors Task Transition, Mode Transition, and Response Transition.

Table 1 about here

\section{Results}

\subsection{Release Times}

Means and standard errors are presented in Figure 2. 
Figure 2 about here

\subsubsection{Control analyses}

The correlation between covert and overt trials was significant, $r=.97, p<.001$, suggesting that participants did engage in $\mathrm{MI}$ on the covert trials as intended by our procedure. In addition, a significant congruency effect of $16 \mathrm{~ms}$ was visible for covert responses, $t(51)=2.71, p<.01$.

\subsubsection{Global/effector-specific motor command inhibition and integration of response mode}

A repeated measures ANOVA with the factors Task Transition (task repetition vs. task switch), Mode (overt vs. covert), and Mode Transition (mode repetition vs. mode switch) was conducted. Only the main effect of Task Transition was significant, $F(1,51)=19.81, p<.001, \eta_{p}^{2}=.28$, MSe $=$ 2,105. RTs were shorter for task repetitions $(826 \mathrm{~ms}, S E=51 \mathrm{~ms})$ than for task switches $(846 \mathrm{~ms}, S E=$ $54 \mathrm{~ms})$. All other effects failed to reach statistical significance ( $\left.p^{\prime} s>.05\right)$.

\subsubsection{Action-specific motor command inhibition}

A repeated measures ANOVA with the within-subjects factors Mode (overt vs. covert), Mode Transition (mode repetition vs. mode switch), and Response Transition (repetition vs. switch) was conducted. Neither the main effect of Response Transition nor any interaction involving this factor was significant, all $F^{\prime} s<1.4, p^{\prime} s>.23$.

In sum, the Release Times showed a significant congruency effect for covert responses as well as a significant correlation of overt and covert responses, indicating that participants did engage in MI. However, effects involving Mode, Mode Transition, or Response Transition were not significant. 


\subsection{Inter rest-key intervals}

Means and standard errors of the IRKIs are presented in Figure 3.

Figure 3 about here

\subsubsection{Control Analyses}

The correlation between overt and covert trials was significant, $r=.73, p<.001$. The congruency effect for covert responses amounted to $29 \mathrm{~ms}, t(51)=4.90, p<.001$.

\subsubsection{Global/effector-specific motor command inhibition and integration of response mode}

A repeated measures ANOVA with the factors Task Transition (task repetition vs. task switch), Mode (overt vs. covert), and Mode Transition (mode repetition vs. mode switch) was conducted.

The main effect of Task Transition was significant, $F(1,51)=16.75, p<.001, \eta_{p}^{2}=.25$, MSe $=$ 1,702 . The mean interval length was shorter on task repetitions $(1110 \mathrm{~ms}, S E=56 \mathrm{~ms})$ compared to task switches $(1127 \mathrm{~ms}, S E=56 \mathrm{~ms})$. The main effect of Mode was also significant, $F(1,51)=28.20, p$ $<.001, \eta_{p}^{2}=.36, \mathrm{MSe}=213,558$. The mean interval length was shorter on covert trials $(998 \mathrm{~ms}, \mathrm{SE}=$ $65 \mathrm{~ms})$ than on overt trials $(1239 \mathrm{~ms}, S E=55 \mathrm{~ms})$. The main effect of Mode Transition was not significant, $F<1.7, p>.20$.

The interaction between Mode and Mode Transition was significant, $F(1,51)=25.64, p<$ $.001, \eta_{p}^{2}=.33, \mathrm{MSe}=7,768$. The mean interval length was significantly $(p<.001)$ shorter on overt trials following upon overt trials $(1213 \mathrm{~ms}, S E=53 \mathrm{~ms})$ compared to overt trials following upon covert trials $(1265 \mathrm{~ms}, S E=58)$. In other words, C-O sequences were slower than O-O sequences. In contrast, the mean interval length on covert trials following upon overt trials was significantly $(p<$ $.001)$ shorter ( $981 \mathrm{~ms}, S E=63 \mathrm{~ms}$ ) compared to the mean interval length on covert trials following upon covert trials $(1016 \mathrm{~ms}, S E=67 \mathrm{~ms})$. O-C sequences were thus faster than $\mathrm{C}-\mathrm{C}$ sequences. 
The three-way interaction between Task Transition, Mode, and Mode Transition was significant, $F(1,51)=7.14 p<.05, \eta_{p}^{2}=.12$, MSe $=1$,322. IRKIs were analyzed for both modes separately. For the overt trials, the main effect of Task Transition was significant, $F(1,51)=12.44 p<$ $.001, \eta_{p}^{2}=.20, \mathrm{MSe}=2,093$. IRKIs were shorter for task repetitions $(1228 \mathrm{~ms}, S E=78 \mathrm{~ms})$ than for task switches $(1250 \mathrm{~ms}, S E=78 \mathrm{~ms})$. The main effect of Mode Transition was also significant, $F(1,51)=$ 29.07, $p<.001, \eta_{p}^{2}=.36$, MSe $=4,813$. The mean interval length was shorter on mode repetitions $(1213 \mathrm{~ms}, S E=74 \mathrm{~ms})$ than on mode switches $(1265 \mathrm{~ms}, S E=82 \mathrm{~ms})$. The interaction between Task Transition and Mode Transition was significant, $F(1,51)=5.31 p<.05, \eta_{p}^{2}=.09$, MSe $=1,815$. Switching the task alone elicited a switch cost of $36 \mathrm{~ms}$. Switching only the mode went along with a cost of $66 \mathrm{~ms}$, whereas switching both task and mode resulted in a cost of $74 \mathrm{~ms}$ (i.e., underadditivity).

For the covert trials, the main effect of Task Transition was significant, $F(1,51)=4.32 p<.05$, $\eta_{p}^{2}=.08, \mathrm{MSe}=1,386 . \mathrm{IRKIs}$ were shorter for task repetitions $(993 \mathrm{~ms}, \mathrm{SE}=91 \mathrm{~ms})$ than for task switches $(1004 \mathrm{~ms}, S E=94 \mathrm{~ms})$. The main effect of Mode Transition was also significant, $F(1,51)=$ $9.31, p<.01, \eta_{p}^{2}=.15, \mathrm{MSe}=7,100$. The mean interval length was shorter on mode switches $(981 \mathrm{~ms}$, $S E=90 \mathrm{~ms})$ than on mode repetitions $(1016 \mathrm{~ms}, S E=95 \mathrm{~ms})$. The interaction between Task Transition and Mode Transition was not significant for covert responses, $F \approx 1, p>.31$. On a descriptive level, pure task switches went along with a cost of $5 \mathrm{~ms}$, whereas pure mode switches elicited a benefit of $42 \mathrm{~ms}$. Switching both task and mode went along with a benefit of $25 \mathrm{~ms}$.

\subsubsection{Action-specific motor command inhibition}

A repeated measures ANOVA with the within-subjects factors Mode (overt vs. covert), Mode Transition (mode repetition vs. mode switch), and Response Transition (repetition vs. switch) was conducted.

The interaction of Response Transition and Mode Transition was significant, $F(1,51)=4.03$, $p<.05, \eta_{p}^{2}=.07, \mathrm{MSe}=1320$. Whereas after overt trials a response repetition benefit of $11 \mathrm{~ms}$ 
occurred, this was not the case after covert trials $(-4 \mathrm{~ms})$. In addition, the three-way interaction between Response Transition, Mode Transition and Mode was significant, $F(1,51)=6.79, p<.05, \eta_{p}^{2}$ $=.12, \mathrm{MSe}=2138$ (Figure 4). To further analyze this interaction, separate ANOVAs were run for both modes. For the overt mode, the interaction was significant, $F(1,51)=10.46, p<.01, \eta_{p}^{2}=.17$, MSe $=$ 1790. Whereas for $\mathrm{C}-\mathrm{O}$ sequences, response repetition costs of $18 \mathrm{~ms}$ were visible, a response repetition benefit of $20 \mathrm{~ms}$ occurred for $\mathrm{O}-\mathrm{O}$ sequences. For the covert mode, the interaction between Mode Transition and Response Transition was not significant, $F<1, p>.86$.

Figure 4 about here

In sum, a significant congruency effect for covert trials was visible in IRKIs, as was a significant correlation of covert and overt trials, indicating again that participants did engage in MI. Furthermore, the IRKIs indicated a mode switch cost for the overt mode and a mode switch benefit for the covert mode, indicating that performing responses covertly goes along with motor inhibition. Regarding the question whether the different response modes are integrated into a single task-set, a sub-additive pattern of switching response mode and task was observed for overt responses, suggesting that the overt response mode can be integrated with other task parameters into a single task-set. For the covert trials, an additive pattern of switching response mode and task was obtained, indicating that the covert response mode was not integrated into the task-set. Finally, a responserepetition cost was observed after covert responses, pointing towards the presence of action-specific motor command inhibition. ${ }^{2}$

\footnotetext{
2 In addition, ANOVAs including the factors Task Transition, Mode, Mode Transition, and Response Transition were run. A significant three-way interaction of Task Transition, Mode Transition, and Response Transition was visible for the Inter Rest-Key Intervals, $F(1,51)=5.89, p<.05, \eta_{p}^{2}=.10$, Mse $=3,144$ : A response repetition benefit of $29 \mathrm{~ms}$ was observed for the task repetition/mode repetition condition, whereas in the other conditions, response repetition effects (benefits as well as costs) were not significant. This can be interpreted in line with the hybrid account proposed by Koch, Frings, and Schuch (2017). However, due to the additional Task Transition Factor, the number of observations per cell dropped by $50 \%$. As a consequence, the statistical power necessary to find the three-way interaction of Mode, Mode Transition, and Response Transition was lost. Because this analysis is important for the main findings of the present study, and in the light of the many analyses already included, the four-way ANOVA is not included in the main body.
} 


\subsection{Additional measures on overt trials}

Means and standard errors for reaction times and error rates of overt trials are presented in Table 1.

Table 1 about here

\subsubsection{Reaction Times}

A repeated measures ANOVA with the factors Task Transition (task repetition vs. task switch), Mode Transition (mode repetition vs. mode switch), and Response Transition (response repetition vs. response switch) was conducted. A main effect of Task Transition could be observed, $F(1,51)=$ $52.52, p<.001, \eta_{p}^{2}=.51, \mathrm{MSe}=4,723$, indicating longer Reaction Times on task switches (1728ms, SE $=35 \mathrm{~ms})$ compared to task repetitions $(1680 \mathrm{~ms}, S E=33 \mathrm{~ms})$. The main effect of Mode Transition was also significant, $F(1,51)=20.88, p<.001, \eta_{p}^{2}=29$, Mse $=8,571$ : mode repetitions went along with faster Reaction Times $(1683 \mathrm{~ms}, S E=33 \mathrm{~ms})$ compared to mode switches $(1725 \mathrm{~ms}, S E=34 \mathrm{~ms})$. Both factors interacted, $F(1,51)=15.84, p<.001, \eta_{p}^{2}=.24$, Mse $=5,638$. This effect was due to complete repetitions ( $1644 \mathrm{~ms}, S E=32 \mathrm{~ms})$ being significantly $\left(p^{\prime} s<.001\right)$ faster than all other conditions $(1722 \mathrm{~ms}, S E=36 \mathrm{~ms}$, for pure task switches, $1715 \mathrm{~ms}, S E=35 \mathrm{~ms}$, for pure mode switches, and $1734 \mathrm{~ms}, S E=34 \mathrm{~ms}$, for double switches), while no other pairwise comparison reached statistical significance $\left(p^{\prime} s>.39\right)$. This pattern is clearly under-additive, the cost of double switches being 59ms smaller than the sum of both types of single switches.

Regarding effects of action-specific motor command inhibition, the interaction of Mode Transition and Response Transition was significant, $F(1,51)=8.28, p<.01, \eta_{p}^{2}=.14$, Mse $=4,737$. For mode repetitions, a response repetition benefit of $20 \mathrm{~ms}$ occurred. For mode switches, on the contrary, a response repetition cost of $19 \mathrm{~ms}$ occurred. The interaction of Task Transition and 
Response Transition was marginally significant, $F(1,51)=3.82, p<.06, \eta_{p}^{2}=.07$, Mse $=4,740$, indicating he usual effect of a response repetition benefit (13ms) for task repetitions and a response repetition cost for task switches (13ms).

\subsubsection{Error Rates}

A repeated measures ANOVA with the factors Task Transition (task repetition vs. task switch), Mode Transition (mode repetition vs. mode switch), and Response Transition (response repetition vs. response switch) was conducted. A significant error switch cost of $0.4 \%$ occurred, $F(1,51)=4.07, p<$ $.05, \eta_{p}^{2}=.07, \mathrm{Mse}=0.00034$. Task Transition and Mode Transition interacted, $F(1,51)=7.97, p<.01$, $\eta_{p}^{2}=.14$, Mse $=0.00032$. Switching the task alone led to a significant cost of $0.9 \%(p<.01)$. Switching the mode alone led to a significant cost of $0.8 \%(p<.05)$. The cost of switching both the task and the mode amounted to $0.7 \%$ and was marginally significant $(p<.06)$.

Regarding action-specific motor command inhibition, none of the effects involving the Response Transition factor reached significance, all $F^{\prime} s<2.4$, all $p^{\prime} s>13$.

\section{Discussion}

Previous research by Rieger et al. (2017) offered evidence for the presence of global and effector-specific motor command inhibition during MI. Nevertheless, during MI participants know in advance that $\mathrm{MI}$ will ensue. In addition, recent findings indicate that (response) inhibition also includes preparatory activity (Elchlepp et al., 2016; Verbruggen et al., 2014). Accordingly, we investigated whether the demand to perform covert responses can be proactively integrated with other task parameters during $\mathrm{Ml}$ and, more specifically, whether task-specific action rules are created during MI. To this end, we used a task-switching procedure, which required participants to switch between two choice-reaction tasks as well as two response modes (overt, covert). 
In a first step, we tested for the presence of indices of motor command inhibition during MI. We observed a response-mode switch cost for the overt response mode $(\mathrm{C}-\mathrm{O}>\mathrm{O}-\mathrm{O})$ and a responsemode switch benefit for the covert response mode (O-C < C-C). In line with Rieger et al. (2017), we thus conclude that when $\mathrm{MI}$ is required on trial $\mathrm{n}-1$, motor-command inhibition takes place that persists over time and affects performance on trial $n$. In contrast, no such inhibition is triggered during overt responding. As noted in the Introduction, the same effector was used during our experiment. Accordingly, we could not dissociate between global- or effector-specific motorcommand inhibition. Yet, our design enabled us to dissociate between response repetitions and response alternations. Because only one effector was used response repetitions were thus action repetitions, whereas responses alternations were action switches. A response-repetition benefit could be observed for O-O sequences of the Inter Rest-Key Intervals that turned into a cost as soon as the trial sequence involved a covert trial. This finding indicates the presence of action-specific motor command inhibition during $\mathrm{Ml}$ and is at odds with the findings of Rieger et al. (2017). We discuss this and another notable difference later on.

In a second step, we investigated the creation of task-specific action rules, which integrate decision and response-mode parameters. For the overt trials, the cost of changing the task or the response mode alone was in the same range as the cost of changing both components simultaneously. Such under-additive pattern of findings replicates the results of Philipp and Koch (2010) and suggests that the overt response mode can be integrated with other task parameters into a single task-set (see also Vandierendonck et al., 2008). In other words, task-specific action rules were created, which combined response mode and task rules (e.g., "if smaller than 5, press the leftkey). For the covert trials, changing the task led to a performance cost and changing the response mode led to a performance benefit. However, both types of transitions did not affect each other. In other words, an additive pattern was observed, which suggests that response-mode switching and task switching occurred independently and that for the covert response mode no task-specific action rules were created. 
The present results suggest that overt and covert responding are underlain by different processes. For the overt response mode, task-specific action rules are created, which incorporate both response-mode information and task-rule information (e.g., "if smaller than 5, press the left-key overtly"). In contrast, for the covert response mode, no mode specific information seems to be integrated. In other words, task-specific action rules such as "if smaller than 5, press the left-key covertly" are not created when participants are cued to perform a task covertly via MI. The presence of a task-switch cost and a response-congruency effect on the covert trials, however, indicates that when a task is performed covertly, its corresponding decision rules are activated. Accordingly, when participants are cued to perform a particular task covertly, two processes are triggered in parallel. First, task-rules are configured, which do not contain response-mode specific information (e.g., "if smaller than 5, left"). Second, motor command inhibition is invoked, which avoids the overt execution of a response.

Regarding the observation that task-specific action rules are restricted to the overt response mode, a possible explanation may be that the integration of mode-specific and task-specific information only serves the overt execution of a response. More specifically, task-specific action rules may be created in order to prepare the cognitive system for an optimal overt application of a task. In contrast, during $\mathrm{MI}$ a task needs to be simulated and overt execution avoided. Creating taskspecific action rules, which include actions that can be performed overtly (e.g., press a left key) together with the connotation that these actions should in fact not be performed overtly, may not be an optimal strategy during MI. Within this view, task-specific action rules are thus not constructed during $\mathrm{Ml}$ in order to avoid overt responses. Alternatively, it could be that it is simply not possible or at least in the present context - to create task-specific action rules for covert actions. Indeed, previous research by Philipp, Jolicoeur, Falkenstein, and Koch (2007) suggested that overt response execution is needed to further integrate all elements of a task-set and thus create task-specific action rules $^{3}$. These authors manipulated the timing of the onset of a go or no-go signal in a task-switching

\footnotetext{
${ }^{3}$ We thank Iring Koch for directing our attention to this possibility.
} 
paradigm in which participants were not allowed to respond until a go-signal was presented. They found that increasing the delay of the signal, thus providing more time for response preparation, facilitates performance strongly if the trial turns out to be a go trial, but if it turned out to be a no-go trial, no after-effects of task-specific response preparation could be found, even though participants must have prepared the task and the response to the same degree in both conditions, because the nature of the signal (i.e., go vs. no-go) was not predictable. These findings suggest that some of the relevant bindings that create persistent aftereffects require actual response execution and not only preparation to execute. Accordingly, the present results show that even covertly responding to a stimulus is not sufficient to integrate all elements of task-set and create task-specific action rules. This conclusion is also in line with recent research on the creation of task-sets on the mere basis of instructions (see Brass, Liefooghe, Braem, \& De Houwer, 2017; Meiran, Liefooghe, \& De Houwer, 2017). Whereas initial task-sets can be formed on the basis of instructions (e.g., Liefooghe, Wenke, \& De Houwer, 2012; Meiran et al., 2015), these task-sets do not integrate all instructed information and practice is needed to incorporate these additional instructions (see Braem et al., 2017 for a demonstration in the domain of context-specific automaticity). Taken together, the present results corroborate with previous findings and indicate that overt task execution has an added value in shaping task-set representations, which can possibly not be obtained on the basis of $\mathrm{MI}$ (see also Theeuwes et al., in press for a similar conclusion).

There are two notable differences between the present findings and the results of Rieger et al. (2017), which need to be discussed. First, as mentioned earlier on, we did obtain evidence for action-specific motor inhibition, whereas this was not the case in the study of Rieger et al. (2017). One possible explanation for this difference is based on the response-discrimination account of Ansorge and Wühr (2004; see also Hommel, 2009). This account states that actions are discriminated by using the perceptual effects that distinguish between these actions. In the present study, only one effector (the right hand) was used and the salient discriminative feature was a movement to either the left-sided or to the right-sided response. Motor command inhibition could thus have operated at the level of this salient feature, leading to action-specific motor command inhibition. The specific 
response display used in the present study could in addition have inflated action-specific motor command inhibition on the basis of spatial positive and negative response priming. In the study of Rieger and colleagues (2017), two effectors (the left and the right hand) were used. Accordingly, in the study of Rieger et al. (2017) participants had to discriminate between two response hands and two possible actions within each hand. Possibly, in this case the discrimination between hands was more salient than the discrimination between specific actions within each hand. Accordingly, motor command inhibition became effector specific rather than action specific. Taken together, the difference in results between both studies may suggest that motor command inhibition is bound to the most salient discriminative feature in a task.

A second main difference between the present study and the study of Rieger et al. (2017) is that, in the present study, IRKIs were generally faster for covert than for overt responses, whereas the opposite pattern was observed in the study of Rieger et al. (2017). At a first glance, this could be taken as evidence that participants in the present study did not engage in $\mathrm{Ml}$ as thoroughly as in the study of Rieger et al. (2017), especially since the general MI ability of participants was not assessed with $\mathrm{MI}$ questionnaires. The reason for this is that no valid MI questionnaires are available in Dutch. Besides, it should be noted that no correlation between behavioral data and MI questionnaires have been expected, which is due to the fact that $\mathrm{MI}$ ability seems to be highly dependent on the specific movement to be imagined (Rieger, personal communication, 24.07.2017). As a consequence, no correlation between the movements in the present experiment (i.e., keystrokes) and movements typically included in MI questionnaires (e.g., walking or cycling) was assumed. However, the correlations between overt and covert movements were extremely high and we furthermore observed a task-rule congruency effect on the covert trials. Both findings thus indicate that participants did engage in $\mathrm{MI}$ as we intended. Furthermore, IRKIs on the covert trials of the present study were one average $998 \mathrm{~ms}$ and on average $933 \mathrm{~ms}$ in the study of Rieger et al. (2017). In contrast, for the overt trials IRKIs were on average $1239 \mathrm{~ms}$ in the present study and $627 \mathrm{~ms}$ in the study of Rieger et al. (2017). The difference between both studies thus mainly resides in a difference in 
performance on the overt trials. One possible interpretation for this difference is that in the present study participants had to switch between two sets of arbitrary category-to-response mappings (e.g., odd -> left), whereas in the study of Rieger et al. (2017) participants performed only one task, in which they had to apply spatially compatible stimulus-response mappings (e.g., outer left circle -> outer left response). Our tasks may thus have been much more difficult for participants to perform overtly compared to the task used by Rieger and colleagues. This difference in performance between both studies may then have been levelled out on the covert trials. Alternatively, it is also possible that observing similar IRKIs on the covert trials of both studies is a coincidence. Within this view, the possibility arises that the spatially compatible stimulus-response mappings used by Rieger et al. (2017) induced additional inhibition on the covert trials. It is reasonable to assume that applying spatially compatible stimulus-response mappings is to a large degree automatic. As such, during MI the automatic tendency to respond needs to be additionally suppressed, which can lead to a general increase in response time. Such automaticity did not come into play in the present study, as the tasks were much more arbitrary.

To conclude, we need to consider the present results in light of the MST (Jeannerod, 1994, 2001, 2006). Our findings indicate that even if participants know in advance that a covert response is needed, they do not create task-specific action rules for MI. This could indicate that the demand to perform an action covertly is not part of the imagery experience and thus not integrated within the construction of the mental representation of the imagined action (cfr. Guillot et al., 2012a). The observation of markers of motor-command inhibition, may furthermore lead to the conclusion that motor-commands in $\mathrm{Ml}$ are not subthreshold in nature. However, some nuance is needed. First, if the covert response mode is not integrated with the other task parameters, what mental representation is responsible for triggering motor-command inhibition? Research underlying the importance of proactive control in response inhibition (e.g., Elchlepp et al., 2016; Verbruggen et al., 2014), indicates that besides response parameters also attentional parameters are adapted to prepare for the eventual inhibition of a response. Accordingly, the present results do not exclude 
that the demand to respond covertly is represented at a different level than the decision rules of a task. Second, the observation of motor-command inhibition does not undermine the hypothesis that overt responses during $\mathrm{MI}$ can also be avoided by means of subthreshold signals. In the current context it seems that overt responses are mainly avoided by means of motor-command inhibition. The demand to frequently switch between response modalities, however, may have increased the need for motor-command inhibition and somewhat overshadowed the role of subthreshold signals. In other words, the pathway by which overt actions are avoided in $\mathrm{MI}$, may depend on the specific task context.

\section{Conflict of interest:}

The authors declare that they have no conflict of interest.

\section{Ethical Standards:}

The present study was performed in accordance with the ethical standards laid down the Declaration of Helsinki and approved by the Ethical Committee of Ghent University. 


\section{References}

Allport, A., Styles, E. A., \& Hsieh, S. (1994). Shifting intentional set: Exploring the dynamic control of tasks. In C. Umilta \& M. Moscovitch (Eds.), Conscious and nonconscious information processing: Attention and performance XV (S. 421-452). Cambridge, MA: MIT Press.

Ansorge, U., \& Wühr, P. (2004). A response-discrimination account of the Simon effect. Journal of Experimental Psychology: Human Perception and Performance, 30(2), 365-377. 
Burianová, H., Marstaller, L., Sowman, P., Tesan, G., Rich, A. N., Williams, M., Savage, G., \& Johnson, B. W. (2013). Multimodal functional imaging of motor imagery using a novel paradigm. Neurolmage, 71, 50-58. DOI: 10.1016/j.neuroimage.2013.01.001.

Decety, J., \& Grèzes, J. (1999). Neural mechanisms subserving the perception of human actions. Trends in Cognitive Sciences, 3(5), 172-178.

Decety, J., Jeannerod, M., \& Prablanc, C. (1989). The timing of mentally represented actions. Behavioural Brain Research, 34(1), 35-42.

Dreisbach, G., \& Haider, H. (2009). How task representations guide attention: further evidence for the shielding function of task sets. Journal of Experimental Psychology: Learning, Memory, and Cognition, 35(2), 477-486.

Elchlepp, H., Lavric, A., Chambers, C. D., \& Verbruggen, F. (2016). Proactive inhibitory control: A general biasing account. Cognitive Psychology, 86, 27-61.

Guillot, A., \& Collet, C. (Eds.). (2010). The neurophysiological foundations of mental and motor imagery. Oxford University Press.

Guillot, A., Di Rienzo, F., MacIntyre, T., Moran, A., \& Collet, C. (2012a). Imagining is not doing but involves specific motor commands: A review of experimental data related to motor inhibition. Frontiers in Human Neuroscience, 6, 247.

Guillot, A., Hoyek, N., Louis, M., \& Collet, C. (2012b). Understanding the timing of motor imagery: Recent findings and future directions. International Review of Sport and Exercise Psychology, 5, 3-22. DOI: 10.1080/1750984X.2011.623787.

Highben, Z., \& Palmer, C. (2004). Effects of auditory and motor mental practice in memorized piano performance. Bulletin of the Council for Research in Music Education, 58-65. 
Hommel, B. (2009). Action control according to TEC (theory of event coding). Psychological Research, 73, 512-526.

Hübner, R., Futterer, T., \& Steinhauser, M. (2001). On attentional control as a source of residual shift costs: Evidence from two-component task shifts. Journal of Experimental Psychology: Learning, Memory, and Cognition, 27(3), 640-653.

Jeannerod, M. (1994). The representing brain: Neural correlates of motor intention and imagery. Behavioral and Brain Sciences, 17(2), 187-202.

Jeannerod, M. (2001). Neural simulation of action: A unifying mechanism for motor control. Neurolmage, 14, S103-S109.

Jeannerod, M. (2004). Actions from within. International Journal of Sport and Exercise Psychology, 2(4), 376-402.

Jeannerod, M. (2006). Motor cognition: What actions tell the self (No. 42). Oxford University Press.

Kiesel, A., Steinhauser, M., Wendt, M., Falkenstein, M., Jost, K., Philipp, A. M., \& Koch, I. (2010). Control and interference in task switching - a review. Psychological Bulletin, 136, 849-874.

Kleinsorge, T. (1999). Response repetition benefits and costs. Acta Psychologica, 103(3), 295310.

Kleinsorge, T., \& Heuer, H. (1999). Hierarchical switching in a multi-dimensional task space. Psychological Research, 62(4), 300-312.

Koch, I., Frings, C., \& Schuch, S. (2017). Explaining response-repetition effects in task switching: Evidence from switching cue modality suggests episodic binding and response inhibition. Psychological Research, https://doi.org/10.1007/s00426-017-0847-9. 
Kraeutner, S., Gionfriddo, A., Bardouille, T., \& Boe, S. (2014). Motor imagery-based brain activity parallels that of motor execution: Evidence from magnetic source imaging of cortical oscillations. Brain Research, 1588, 81-91. DOI: 10.1016/j.brainres.2014.09.001.

Leys, C., Ley, C., Klein, O., Bernard, P., \& Licata, L. (2013). Detecting outliers: Do not use standard deviation around the mean, use absolute deviation around the median. Journal of Experimental Social Psychology, 49, 764-766. DOI: 10.1016/j.jesp.2013.03.013.

Lim, S., \& Lippman, L. G. (1991). Mental practice and memorization of piano music. The Journal of General Psychology, 118(1), 21-30.

McAvinue, L. P., \& Robertson, I. H. (2008). Measuring motor imagery ability: a review. European Journal of Cognitive Psychology, 20(2), 232-251.

Meiran, N., \& Kessler, Y. (2008). The task rule congruency effect in task switching reflects activated long-term memory. Journal of Experimental Psychology: Human Perception and Performance, 34, 137-157. DOI: 10.1037/0096-1523.34.1.137.

Monsell, S. (2003). Task switching. Trends in Cognitive Sciences, 7(3), 134-140.

Monsell, S., \& Mizon, G. A. (2006). Can the task-cuing paradigm measure an endogenous task-set reconfiguration process? Journal of Experimental Psychology: Human Perception and Performance, 32, 493-516. DOI: 10.1037/0096-1523.32.3.493.

Moran, A., Guillot, A., Maclntyre, T., \& Collet, C. (2012). Re-imagining motor imagery: Building bridges between cognitive neuroscience and sport psychology. British Journal of Psychology, 103(2), 224-247.

Nyberg, L., Eriksson, J., Larsson, A., \& Marklund, P. (2006). Learning by doing versus learning by thinking: an fMRI study of motor and mental training. Neuropsychologia, 44(5), 711-717. 
Philipp, A. M., Jolicoeur, P., Falkenstein, M., \& Koch, I. (2007). Response selection and response execution in task switching: Evidence from a go-signal paradigm. Journal of Experimental Psychology: Learning, Memory, and Cognition, 33(6), 1062-1075.

Philipp, A. M., \& Koch, I. (2010). The integration of task-set components into cognitive task representations. Psychologica Belgica, 50(3-4), 838-411.

Rieger, M., Dahm, S. F., \& Koch, I. (2017). Inhibition in motor imagery: A novel action mode switching paradigm. Psychonomic Bulletin \& Review, 24(2), 459-466.

Rieger, M., \& Gauggel, S. (1999). Inhibitory after-effects in the stop signal paradigm. British Journal of Psychology, 90(4), 509-518.

Rogers, R. G. (2006). Mental practice and acquisition of motor skills: examples from sports training and surgical education. Obstetrics and Gynecology Clinics of North America, 33(2), 297-304.

Rogers, R. D., \& Monsell, S. (1995). Costs of a predictable switch between simple cognitive tasks. Journal of Experimental Psychology: General, 124, 207-231.

Sudevan, P., \& Taylor, D. A. (1987). The cueing and priming of cognitive operations. Journal of Experimental Psychology: Human Perception and Performance, 13, 89-103. DOI: 10.1037/00961523.13.1.89.

Vandierendonck, A., Christiaens, E., \& Liefooghe, B. (2008). On the representation of task information in task switching: Evidence from task and dimension switching. Memory \& Cognition, 36(7), 1248-1261.

Vandierendonck, A., Liefooghe, B., \& Verbruggen, F. (2010). Task switching: interplay of reconfiguration and interference control. Psychological Bulletin, 136(4), 601-626.

Verbruggen, F., \& Logan, G. D. (2008). Response inhibition in the stop-signal paradigm. Trends in Cognitive Sciences, 12(11), 418-424. 
Verbruggen, F., \& Logan, G. D. (2009). Models of response inhibition in the stop-signal and stop-change paradigms. Neuroscience \& Biobehavioral Reviews, 33(5), 647-661.

Verbruggen, F., Logan, G. D., Liefooghe, B., \& Vandierendonck, A. (2008). Short-term aftereffects of response inhibition: Repetition priming or between-trial control adjustments? Journal of Experimental Psychology: Human Perception and Performance, 34(2), 413-426.

Verbruggen, F., Stevens, T., \& Chambers, C. D. (2014). Proactive and reactive stopping when distracted: An attentional account. Journal of Experimental Psychology: Human Perception and Performance, 40(4), 1295-1300.

Weinberg, R. (2008). Does imagery work? Effects on performance and mental skills. Journal of Imagery Research in Sport and Physical Activity, 3(1), 1-21.

\begin{tabular}{llcrrrr} 
& & \multicolumn{2}{c}{ Mode Repetition } & Mode Switch \\
\cline { 3 - 6 } & & Response & Response & Response & Response & M \\
& & Repetition & Switch & Repetition & Switch & \\
\hline \multirow{2}{*}{ Reaction } & Task Repetition & $1629(33)$ & $1660(32)$ & $1717(35)$ & $1713(35)$ & $1680(33)$ \\
Times & Task Switch & $1718(35)$ & $1726(38)$ & $1752(35)$ & $1717(35)$ & $1728(35)$ \\
& M & $1673(33)$ & $1693(35)$ & $1734(34)$ & $1715(35)$ & \\
& & & & & & \\
Error & Task Repetition & $0.8(0.2)$ & $0.9(0.2)$ & $2.0(0.4)$ & $1.4(0.3)$ & $1.3(0.1)$ \\
Rates & Task Switch & $1.6(0.3)$ & $1.9(0.4)$ & $1.6(0.4)$ & $1.6(0.3)$ & $1.7(0.2)$ \\
& M & $1.2(0.2)$ & $1.4(0.3)$ & $1.8(0.2)$ & $1.5(0.2)$ &
\end{tabular}


Table 1. Mean reaction times [ms] and error rates [\%] of overt trials as a function of Task Transition, Mode Transition, and Response Transition. SEM are given in parentheses.

Figure Captions:

Figure 1. Overview of the procedure of single trials.

Figure 2. Mean Release Timess [ms] as a function of Mode (Overt vs. Covert), Mode Transition (Mode Repetition vs. Mode Switch), and Task Transition (Task Repetition vs. Task Switch). Error bars represent SEM.

Figure 3. Mean Inter Rest-Key Intervals [ms] as a function of Mode (Overt vs. Covert), Mode Transition (Mode Repetition vs. Mode Switch), and Task Transition (Task Repetition vs. Task Switch). Error bars represent SEM. 
Figure 4. Mean Inter Rest-Key Intervals [ms] as a function of Mode (Overt vs. Covert), Mode Transition (Mode Repetition vs. Mode Switch), and Response Transition (Response Repetition vs. Response Switch). Error bars represent SEM.

Covert

Trials

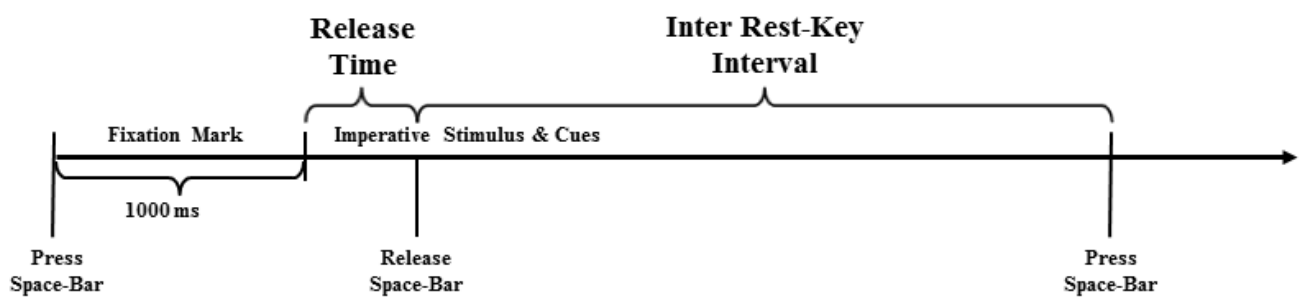

Overt

Trials

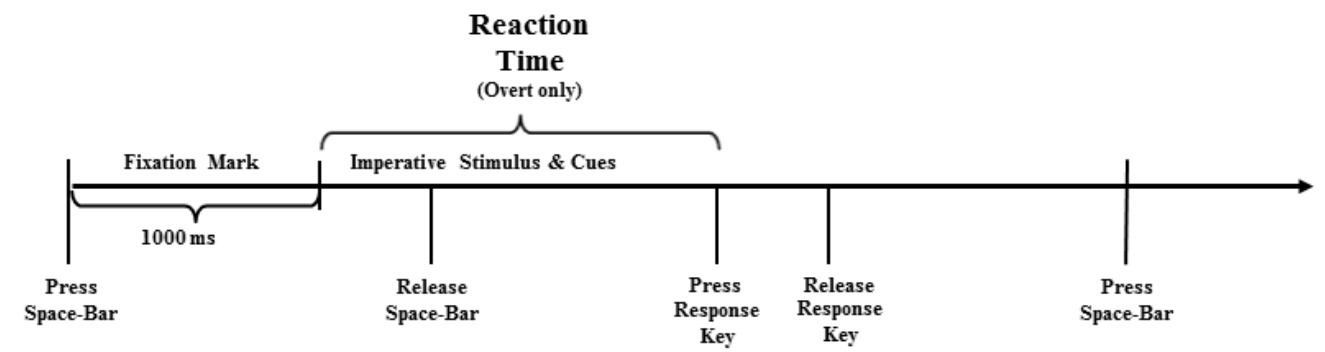


Figure 1 


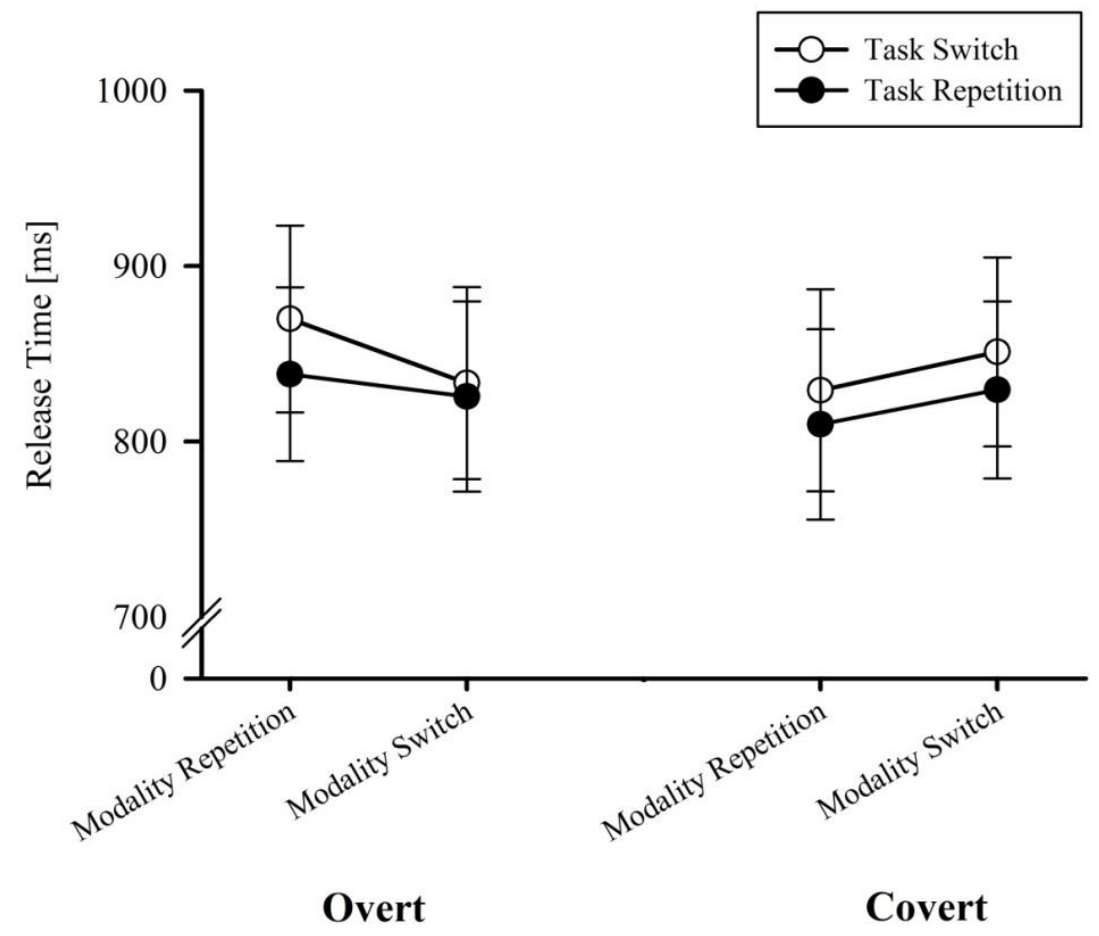

Figure 2 


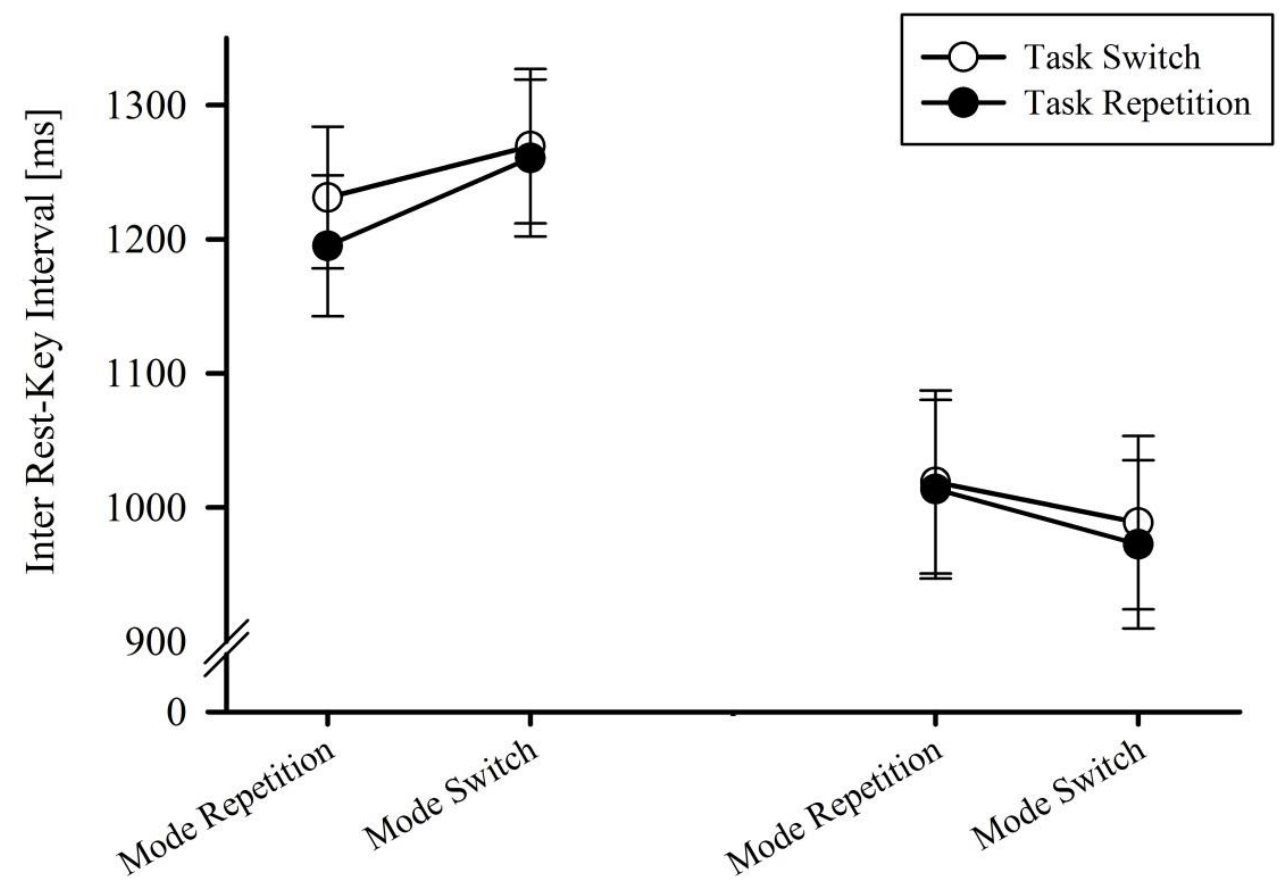

Overt

Covert

Figure 3 


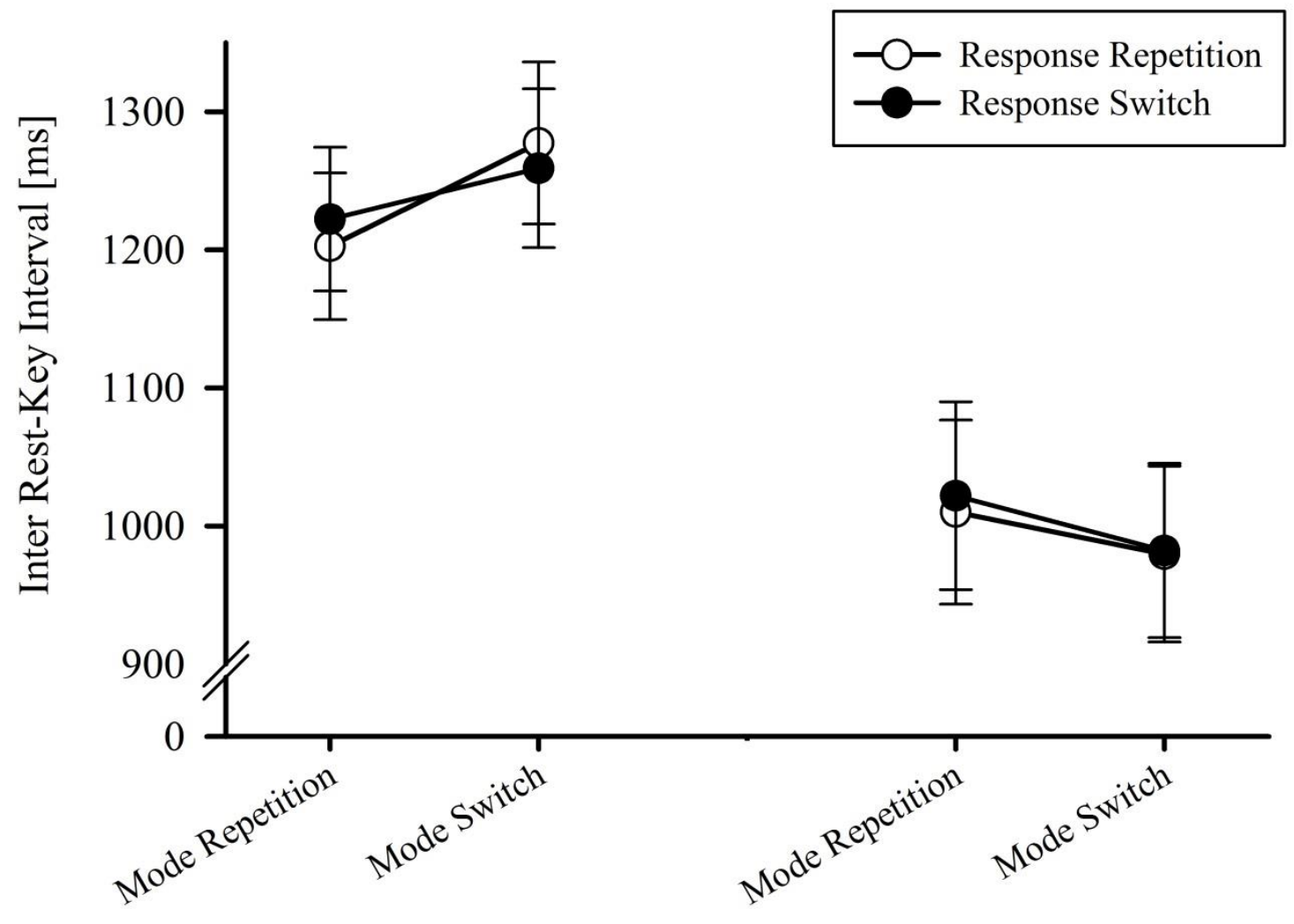

Overt

Covert

Figure 4 